\title{
Comparison of Food Consumption Score (FCS) and Calorie Intake Indicators to Measure Food Security*
}

\author{
Jiaqi HUANG, Fengying NIE* \& Jieying BI \\ Agricultural Information Institute, Chinese Academy of Agricultural Sciences, Beijing, China
}

*Corresponding author: Prof. Fengying Nie, E-mail: niefengying@caas.cn

\begin{abstract}
This study summarized international common used indicators to measure food security, and compared two indicators, calorie intake and Food Consumption Score (FCS) based on the evidence of filed survey in 6 rural counties of west China. The results showed that the proportion of food secure households is $75.8 \%$ measured by the calorie intake, which are nearly 10 percentage points lower than that of measured by FCS. The match degree of the results measured by these two indicators is $70.03 \%$. FCS indicator narrows down the gap of grain contributions and enlarges the gap of poultry and meats contributions compare with the calorie indicator. FCS considers diet diversity and balance more.
\end{abstract}

KEYWORD: Food consumption score; Calorie intake; Food security

\section{INTRODUCTION}

With the high speed of economic growth in China, living standard of the Chinese people has greatly improved. From 2000 to 2013, the per capita GDP of China rose from 7858 Yuan to 41805 Yuan, per capita net income of rural residents in China increased from 2253 Yuan to 8896 Yuan, and the Engle's coefficient of rural residents in China decreased from $49.1 \%$ to $37.7 \%$ (NBS, 2014). It seems that very few people in rural China still suffer from hunger now. However, according to Food and Agriculture Organization (FAO), there are still 158 million undernourished people in China in 20102013 , accounting for $11.4 \%$ of the total population of China and $18.8 \%$ of the world's total undernourished population. This shows that China still requires consistent and sustained work on fighting against hunger and reducing undernourished population.

To reduce hunger and improve the food security situation, the basic question is how many people are food insecure. To answer this, food security assessment or measurement must be clear. However, as the complexity of food security, it is still discussed by many researchers and organizations. In recent years, a growing number of institutions and scholars began to use terminologies of "food and

\footnotetext{
* This study is funded by National Natural Science Foundation of China, project title "Study on food security of rural poor", project No. 71173222.
}

nutrition security" and "food security and nutrition" to emphasis on improving the nutritional level is the ultimate goal of food security.

Food security measurement can be divided into one-dimensional and multi-dimensional categories. One-dimensional indicator mainly including: (1) The FAO Indicator of Undernourishment (FAOIU). It is an indicator of undernourishment for most of the countries and considers mean dietary energy supply as a proxy for food energy consumption. However, many scholars argue that the calorie availability is a poor predictor of food security and nutritional development, and it might also result in a large underestimation of undernutrition (Svedberg, 2000; Qaim, et al., 2007; Babatunde and Qaim, 2010; Dasgupta, 1993); (2) The Diet Diversity Score (DDS). It represents the number of different foods or food groups consumed over a given reference period (Hoddinott and Yohannes 2002). (3) The Food Consumption Score (FCS). It is calculated and summed by the frequency of consumption of each kind of food in 7 days multiplied by the assigned weight (Nie et al., 2011). (4) Anthropometric indicators (AI). AI such as stunting (low height-forage), underweight (low weight-for-age), and wasting (low weight-for-height) measure nutritional outcomes at the individual level (Morris S., 2001). Even though AI measure nutritional outcomes directly, but they do not cover specific nutrients that might be deficient (Walker et al. 2007, Svedberg 2011). (5) Medical and biomarker indicators (MBI). Biochemical indicators can to measure micronutrient 
deficiencies with precision by medical tests, but due to the high cost, this method can hardly be encouraged using in most of the research projects.

The multi-dimensional indicators mainly include: (1) The Global Hunger Index (GHI). The GHI is developed by IFPRI and combines three indicators: undernourishment, child underweight, and child mortality, which is respectively source from FAO, WHO, and UNICEF. Compare with FAOIU, GHI is more comprehensive, but it may have the issue of double counting (Masset 2011). (2) The Global Food Security Index (GFSI). The GFSI is based on a consistent framework and assesses food security across three dimensions: affordability, availability and utilization. Unlike the GHI, the GFSI not only assesses the food security in developing counties but also in developed countries. (3) The Poverty and Hunger Index (PHI). The PHI links poverty and hunger or nutrition together, the indicators for measuring nutritional dimension is the prevalence of children underweight, and the proportion of undernourished population (Gentilini and Webb, 2008). (4) The Hunger Reduction Commitment Index (HRCI). Unlike the previous indicators emphasize the outputs of food and nutrition security, the HRCI is a major attempt to assess the governance and political commitment to reduce undernutrition (Lintelo 2012).

Among these indicators, calorie intake or calorie availability and Food Consumption Score (FCS) are most common used by researchers. Based on evidence of filed survey in 6 rural counties of west China, this study will compare the results measured by calorie intake and FCS, and try to summarize their differences, advantages and disadvantages.

\section{DATA AND INDICATORS}

\subsection{Data}

The data used in this study was obtained from the household survey in 6 poor rural counties in west China, which are Zhen'an and Luonan of Shaanxi Province, Wuding and Huize of Yunnan Province, and Pan and Zheng'an of Guizhou Province. These counties were selected randomly from a group which concludes the poorest counties in China. The sample size was calculated according to the standard calculation formula:

$$
\mathrm{n}=\operatorname{DEFF} \times \frac{\left(\mathrm{Z}_{\alpha / 2}\right)^{2} \times[\mathrm{p} \times(1-\mathrm{p})]}{\mathrm{d}^{2}}
$$

Where in, $\mathrm{n}$ represents the calculated sample size; DEFF is design effect; $Z_{\alpha / 2}$ is the threshold; $\alpha$ is significance level; $1-\alpha$ is confidence level; $p$ for the estimated prevalence of food insecurity; $\mathrm{d}$ is precision.
A response rate of 0.9 was used, under the assumption that not all the households would participate in the survey. This is to say that households in each county were oversampled by around $10 \%$. Other technical indicators related to sampling include: $95 \%$ of confidence level, $50 \%$ of estimated prevalence of food security, precision of $10 \%$ and a design effect of 2 . The calculated sample size for one county was 214 , we selected 12 households per village according to former survey experience, and sampled 19 villages in each county, so the actual sample size $n=228$ for each county and the total sample size for 6 counties was $\mathrm{N}=1368$.

As to the survey on food consumption, a recall method is used. For the exact food consumption survey for calorie intake calculation, the recall period is a month. Sample counties are located in mountainous areas, where road infrastructure is poor and economy level of development occupies to the backward condition. Various factors caused the monotonousness of food consumption of the households living there. If recall period is too short, zero consumption of many kinds of food of a large number of households would appear. In order to obtain comprehensive data, the past month is selected as the recall period. For the Food Consumption Score (FCS) calculation, the standard recall period is the past 7 days.

\subsection{Indicators Calorie Intake}

World Food Programme (WFP) recommends FCS, and calorie intake as well to measure food security (WFP, 2005). WFP suggests three cut points derived from a basic dietary energy requirement of approximately 2100 Kcal per capita per day, corresponding to shortfalls of 0,10 , and 30 percent relative to requirements. If the calorie consumption is less than $1470 \mathrm{Kcal}$ per capita per day, then it considered in poor food security situation (Table 2).

Due to the similar food security group definition by WFP in FCS and calorie intakes (poor, borderline, and acceptable group), and in order to compare the results measured by these two indicators, this study follows the WFP calorie intakes and FCS grouping thresholds (Table 2).

For calculating the calorie intake, the first step is to convert the food consumption into calorie intake. In this regard, the following two points should be noted:

First, the tool using for converting is Food Composition Table (FCT). However, the FCT of different countries are also different, FCT of China developed by Chinese Center for Disease Control and Prevention National Institute for Nutrition and Food Safety was used in this study.

Second, the food consumed including food consumed at home and food consumed away from 
home. For the food consumed at home, the consumption of each commodity (such as rice, wheat flour, vegetables, pork, etc.) was recorded in the questionnaire. But for the food consumed away from home, only the expenditure was recorded. So, the expenditure must be converted into calorie inkate. In this regard, FAO and WFP both use calorie price (FAO Statistics Division, 2012), which can be calculated by the total food expenditures occurred by household acquire the food products consumed at home divided by the total dietary energy of household get from the food consumed at home. Then the calorie intake from food consumed away from home can be calculated by expenditures of food consumed away from home divided by calorie price. However, if calculated in this way, it will obviously overestimate calorie consumption of dinning out. Because of the operating costs of the restaurants, the calorie price of food consumed away from home is much higher than the calorie price of food consumed at home, so before calculating the calorie price, the expenditure of dinning out was reduced by $50 \%$.

Thus, the total calorie intake for a household is:

$$
\begin{aligned}
\mathrm{C}=\sum_{i=1}^{n} C_{\text {rice }} & +C_{\text {flour }}+C_{\text {pork }}+\cdots+C_{\text {food }(n)} \\
& +50 \% \mathrm{E}_{\text {away }} / \mathrm{P}(\mathrm{C})
\end{aligned}
$$

Where $\mathrm{C}$ represents the total calculated calorie intake; $\mathrm{n}$ is total kinds of food items; $\mathrm{C}_{\mathrm{food}(\mathrm{n})}$ is calorie contribution from food $n$; $E_{\text {away }}$ is expenditures of food consumed away from home; $\mathrm{P}(\mathrm{C})$ is calorie price:

$$
\begin{aligned}
\mathrm{P}(\mathrm{C})=\mathrm{E}_{\text {home }} & /\left(\sum_{i=1}^{n} C_{\text {rice }}+C_{\text {flour }}+C_{\text {pork }}+\cdots\right. \\
& \left.+C_{\text {food }(n)}\right)
\end{aligned}
$$

Where, $E_{\text {home }}$ is total food expenditures occurred by household acquire the food products consumed at home.

According to RNI, if the calorie intake level of a household is lower than $2100 \mathrm{Kcal}$ per capita per day, then this household will be grouped as calorie inadequate or food insecurity here in this study.

\section{Food Consumption Score (FCS)}

Households were asked about the food that they had consumed over the past 7 days. The response for each of the foods on the list was simply the number of days. Each food group was given a score of 0 to 7 , depending on the number of days it was eaten. The information gathered on dietary diversity and frequency of consumption was analysed by calculating the Food Consumption Score (FCS).
The FCS was calculated based on the diversity of the households' consumption of 8 food groups. Every group was assigned a weight according to the quality of nutrients that they bring to the diet (Table1). For every household involved in the survey, the FCS was calculated by multiplying each food group frequency by food group weight, and then added together.

Table 1. Food item, group and weight of FCS

\begin{tabular}{|l|l|c|}
\hline Food item & Food group & Weight \\
\hline Rice, wheat, potatoes & $\begin{array}{l}\text { Cereals, tubers and } \\
\text { root crops }\end{array}$ & 2 \\
\hline Beans, peas, nuts and tofu & Pulses & 3 \\
\hline Vegetables & Vegetables & 1 \\
\hline Fruits & Fruit & 1 \\
\hline $\begin{array}{l}\text { Pork, beef, sheep, poultry, } \\
\text { eggs and fish }\end{array}$ & Meat and fish & 4 \\
\hline Milk and other dairy & Milk & 4 \\
\hline Sugar & Sugar & 0.5 \\
\hline Edible oil & Oil & 0.5 \\
\hline
\end{tabular}

Source: Nie et al., (2011).

The maximum value of the FCS is 112 , meaning that every food group was consumed every day for the past 7 days. The calculated scores from the analysis are classified into 3 groups. According to World Food Programme (WFP) standards, a score of 0-21 indicates poor food security, a core of 21.5-35 indicates borderline food security and a score greater than 35 is considered an acceptable food security level (Table 2). Poor and borderline groups are considered as food insecure (Nie et al., 2011).

Table 2. Thresholds for food security groups by calorie intake and FCS

\begin{tabular}{|l|l|l|l|}
\hline & $\begin{array}{l}\text { Food security } \\
\text { group }\end{array}$ & $\begin{array}{l}\text { Calorie intake } \\
\text { (Kcal/capita/day) }\end{array}$ & FCS \\
\hline \multirow{2}{*}{$\begin{array}{l}\text { Food } \\
\text { insecurity }\end{array}$} & Poor & $<1470$ & $0-21$ \\
\cline { 2 - 4 } & Borderline & $1470-2100$ & $21.5-35$ \\
\hline $\begin{array}{l}\text { Food } \\
\text { security }\end{array}$ & Acceptable & $\geq 2100$ & $>35$ \\
\hline
\end{tabular}

Source: WFP (2005).

\section{COMPARISON OF FCS AND CALORIE INTAKE MEASUREMENT}

\subsection{Difference}

The proportion of food secure households is $75.8 \%$ measured by the calorie intake, which are nearly 10 percentage points lower than that of measured by FCS (Figure 1). This result was consistence with that of other comparative studies. For example, IFPRI 
made comparative studies using Burundian households' survey data, the poor food insure households account for $10 \%$ by using FCS, which is $46 \%$ by using the calorie intake indicator. The study did in Haiti also showed the same trend (IFPRI, 2009).

$\ltimes$ Acceptable $\because$ Borderline . Poor

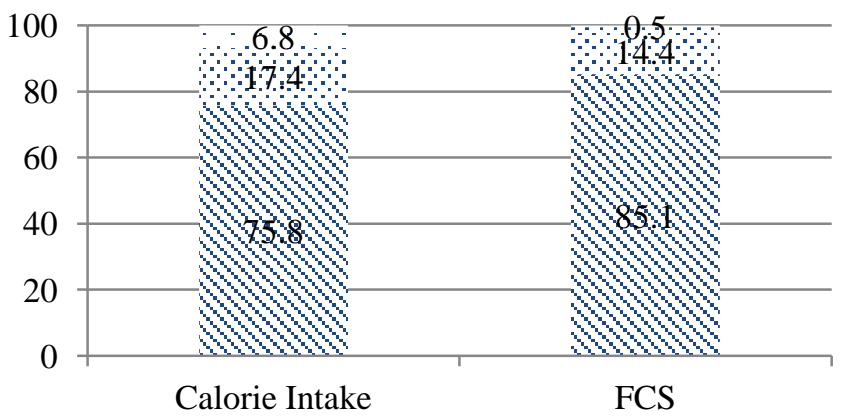

Figure 1. Status of food security grouped by calorie intake and FCS $(\%)$

It seems that FCS overestimates the food security level of households. However, when we did the same comparison county by county, it was found that the "overestimate" situation did not always happen. Luonan is an exception. The proportion of food secured households measured by calorie intake is $84.6 \%$, while that measured by FCS is only $68.0 \%$. When only measuring by calorie intake, we may make a conclusion as Luonan is the most food secure county in the 6 counties. While when we only use FSC to do the measuring, conclusion will be exactly the opposite: Luonan is the most food insecure county (Figure 2).

The results grouped by calorie intake and FCS is quite different. In order to find out the reason for the difference, the calorie contribution from different foods of sample households was further calculated. The food source of calorie mainly comes from grains and tubers, animal foods, and cooking oil. Since the average consumption of grains and tubers, and poultry and meats have significant differences in 6 counties, so the calorie contribution from grains and tubers, and poultry and meats was mainly calculated. The average calorie contribution from grains and tubers of the sample households is $59.8 \%$, but that of Luonan reached $72.5 \%$, which is far beyond the range of $50 \%-60 \%$ recommended by Dietary Guidelines for Chinese Residents. However, the energy contribution from poultry and meats of Luonan is only $1.7 \%$, which indicates that households in Luonan mainly obtain calorie from grains and tubers (Table 3 ). The FCS gives weights to different kinds of food, the weight for grains and tubers is only 2, but the weight for poultry and meats is 4. Households in Luonan consume too little poultry and meats, so they have very low FSC.

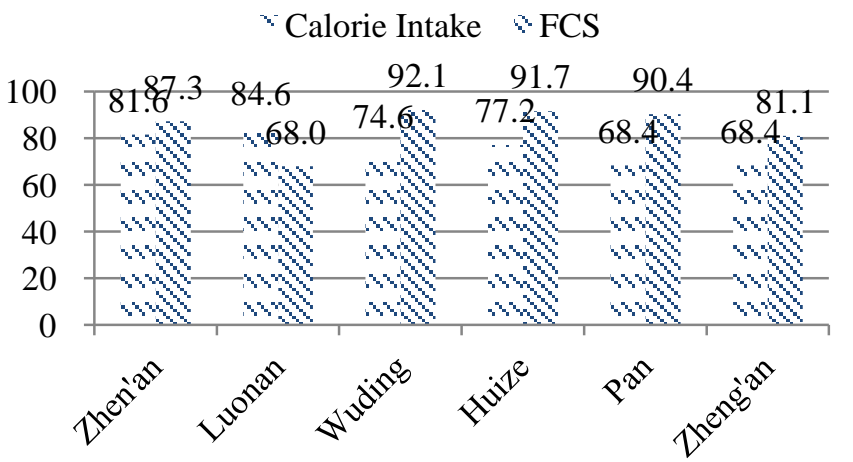

Figure 2. Status of food security grouped by calorie intake and FCS by county (\%)

Table 3. Calorie contribution from foods $(\%)$

\begin{tabular}{|l|c|c|c|c|c|c|c|}
\hline & Zhen'an & Luonan & Wuding & Huize & Pan & Zheng'an & Total \\
\hline Grains and tubers & 59.1 & 72.5 & 55.2 & 53.8 & 58.6 & 59.8 & 59.8 \\
\hline Poultry and meats & 6.9 & 1.7 & 13.3 & 16.6 & 14.1 & 12.1 & 10.8 \\
\hline
\end{tabular}

\subsection{Match degree}

To see how well the classification results for FCS and calorie intake indicators match, a crosstabulation method was applied. It is found that $65.5 \%$ of the households are both food secure measured by these two indicators. However, $19.59 \%$ of the households did not meet the threshold of 2100 Kcal per capita per day, but considered food secure in FCS (Table 4). The match degree of the classification results is $70.03 \%$, which is higher than that in other similar studies (IFPRI, 2009) ${ }^{1}$.

\footnotetext{
1 In this study, the match degree of Burundi was $34.6 \%$, the match degree of Haiti was $57.2 \%$.
}

Table 4. Sample cross-tabulation of FCS and calorie intake groups (\%)

\begin{tabular}{|c|c|c|c|c|}
\hline \multicolumn{2}{|c|}{} & \multicolumn{3}{|c|}{ Calorie intake } \\
\cline { 3 - 5 } \multicolumn{2}{|c|}{} & $\begin{array}{c}\text { Food } \\
\text { secure }\end{array}$ & $\begin{array}{c}\text { Food } \\
\text { insecure }\end{array}$ & Total \\
\hline \multirow{2}{*}{ FCS } & Food secure & 65.50 & 19.59 & 85.09 \\
\cline { 2 - 5 } & Food insecure & 10.38 & 4.53 & 14.91 \\
\hline \multirow{2}{*}{ Total } & & 75.88 & 24.12 & 100.00 \\
\hline
\end{tabular}


Table 5. Match degree (\%)

\begin{tabular}{|l|l|}
\hline Match & 70.03 \\
\hline Poor match & 29.97 \\
\hline
\end{tabular}

\subsection{Reason for poor match}

To further analyse the underlying reason for poor match, and to further find the characters of these poor match households, all sample households were classified into 4 groups (Table 6): both secure (group1), both insecure (group 2), energy secure, FCS insecure (group 3), and FCS secure, energy insecure (group 4).

Table 6. Number of households in different 4 groups* $(\%)$

\begin{tabular}{|c|c|c|c|c|c|}
\hline & Group1 & Group2 & Group3 & Group4 & Total \\
\hline Zhen'an & 168 & 8 & 21 & 31 & 228 \\
\hline Luonan & 132 & 12 & 61 & 23 & 228 \\
\hline Wuding & 162 & 6 & 12 & 48 & 228 \\
\hline Huize & 167 & 8 & 11 & 42 & 228 \\
\hline Pan & 139 & 10 & 12 & 67 & 228 \\
\hline Zheng'an & 128 & 18 & 25 & 57 & 228 \\
\hline Total & 896 & 62 & 142 & 268 & 1368 \\
\hline
\end{tabular}

*Note: both secure (group 1), both insecure (group 2), energy secure, FCS insecure (group 3), FCS secure, energy insecure (group 4)

It is found that 896 of the households are both secure $(65.5 \%), 62$ households are both insecure (4.5\%), 142 households are energy secure but FCS insecure (10.4\%), 268 households are FCS secure but energy insecure (19.6\%). Zhen'an is in the best food security status, because 168 households in Zhen'an are both secure, which is the largest in the 6 counties, and 8 households are both insecure, which is the least. However, Zheng' an is the worst, because it has only 128 households in both secure group, which is the least, and has 18 households in both insecure group, which is the largest. Moreover, for energy secure but FCS insecure group, Luonan has the largest number of households (61), but for FCS secure, energy insecure group, Pan has the largest number of households (67). It has been know that, Luonan consume the largest quantity of food, high calorie, but simple variety of food, imbalanced diet. However, the situation of Pan is just opposite, low calorie intake but relatively balanced diet. Thus, it is not hard to speculate the difference of the food consumption characters between calorie secure FCS insecure group, and FCS secure calorie insecure group.

To further confirm the speculation, the calorie contribution and FCS contribution from each kind of food in each group were calculated (Table 7 and Table 8).
Table 7. Calorie contribution by each kind of food in each group (absolute value)

\begin{tabular}{|c|c|c|c|c|}
\hline & Group 1 & Group2 & Group 3 & Group4 \\
\hline Grains & 2315.2 & 1246.7 & 2899.9 & 1084.1 \\
\hline Beans & 291.2 & 38.3 & 98.2 & 99.8 \\
\hline Vegetables & 45.7 & 24.2 & 47.2 & 27.4 \\
\hline Dairy & 14.5 & 0.0 & 4.0 & 8.1 \\
\hline Fruits & 36.6 & 4.3 & 12.4 & 21.8 \\
\hline Fish \& shrimp & 7.2 & 0.2 & 0.9 & 3.4 \\
\hline Poultry \& meats & 463.5 & 111.9 & 160.8 & 223.2 \\
\hline Eggs & 42.4 & 4.0 & 16.8 & 22.4 \\
\hline Oil & 676.1 & 325.8 & 660.0 & 322.2 \\
\hline Sugar & 52.4 & 9.5 & 37.4 & 18.5 \\
\hline Condiments & 60.7 & 32.3 & 46.1 & 38.3 \\
\hline
\end{tabular}

Table 8. FCS contribution by each kind of food in each group (absolute value)

\begin{tabular}{|l|l|l|l|l|}
\hline & Group 1 & Group2 & Group 3 & Group4 \\
\hline Grains & 32.7 & 30.4 & 32.8 & 29.8 \\
\hline Beans & 11.8 & 1.5 & 2.1 & 9.4 \\
\hline Vegetables & 10.3 & 8.4 & 9.8 & 9.1 \\
\hline Dairy & 4.1 & 0.0 & 0.6 & 3.7 \\
\hline Fruits & 2.6 & 0.3 & 0.3 & 2.4 \\
\hline Fish \& shrimp & 0.9 & 0.0 & 0.1 & 0.6 \\
\hline Poultry \& meats & 17.3 & 2.1 & 2.5 & 15.2 \\
\hline Eggs & 8.7 & 1.0 & 1.4 & 7.0 \\
\hline Oil & 3.5 & 3.2 & 3.3 & 3.4 \\
\hline Sugar & 0.9 & 0.3 & 0.5 & 0.7 \\
\hline Condiments & 0.0 & 0.0 & 0.0 & 0.0 \\
\hline
\end{tabular}

For the calorie contribution, the contribution from each kind of food of both insecure group are all lower than that of both secure group. In energy secure FCS insecure group, the energy contribution from grains is very high, up to $2899.9 \mathrm{Kcal}$, and the contribution from vegetables is $47.2 \mathrm{Kcal}$, which are both higher than those of both secure group. But the energy contribution from dairy, fruits, fish and shrimps, poultry and meats, and eggs of energy secure FCS insecure group are all far lower than those of both secure group, and are also 50.4\%, $43.0 \%, 72.5 \%, 28.0 \%$, and $24.7 \%$ lower than those of FCS secure energy insecure group. It is not difficult to see that, for the energy secure FCS insecure group, calorie is mainly contributed by grains, the energy contributed by other foods is very low, and the diet is imbalanced. So, even though this group of households meets the standard of calorie intake, it cannot meet the food secure threshold of FCS which is concern more with diet diversity. 
However, the situation of FCS secure energy insecure group is exactly the opposite, the calorie contribution from grains is only $1,084.1 \mathrm{Kcal}$, which is low, the calorie contribution from vegetables and oil is also lower than energy secure FCS insecure group, but that from dairy, fruits, fish and shrimps, poultry and meats, and eggs are high.

For the FCS contribution, the contribution from each kind of food of both insecure group are all lower than that of both secure group. In energy secure FCS insecure group, the calorie contribution from grain is very high, up to 32.8 scores, which is even a little bit higher than 32.7 scores in both secure group. The contribution from vegetables is 9.8 scores, which is a little bit higher than that of FCS secure energy insecure group. But the FCS contribution from dairy, fruits, fish and shrimps, poultry and meats, and eggs are far lower than those of both secure group, and is $84.0 \%, 88.8 \%, 77.0 \%$, $83.6 \%$, and $79.8 \%$ lower than those of FCS secure energy insecure group.

The relative proportions of calorie and FCS contribution from each kind of foods of each group were also calculated (Figure 3). For the calorie contribution rate, the grain contribution rate of each group is over $50 \%$, that of both secure group is $57.5 \%$, which is the lowest, while that of energy secure FCS insecure group is $73.3 \%$, which is the highest. The calorie contribution rate from foods is quite different between energy secure FCS insecure group and FCS secure energy insecure group, the grain calorie contribution rate of the former is $73.3 \%$, but that of the latter is only $57.8 \%$, the poultry and meats energy contribution of the former is only $4.1 \%$, but that of the latter is $11.9 \%$.

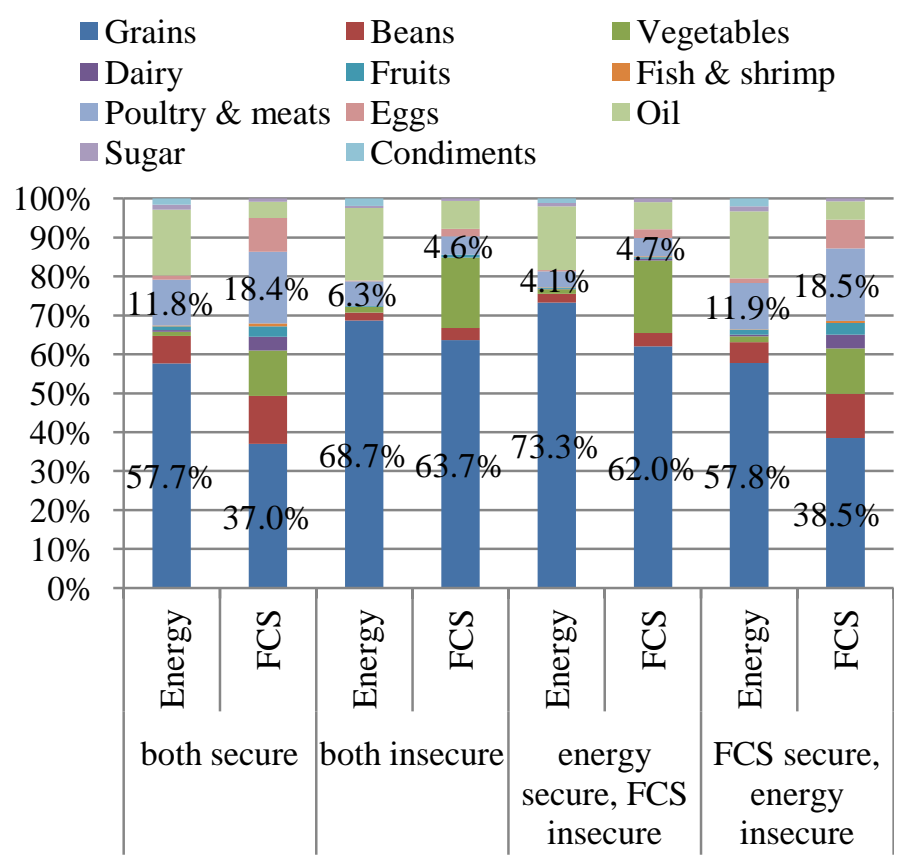

Figure 3. Status of food security grouped by calorie intake and FCS by county $(\%)$
For the FCS contribution rate, compare with FCS insecure groups, the grains contribution rate of FCS secure groups is lower, but the poultry and meats contribution is higher. To be more specific, the grain FCS contribution rate of both secure group and FCS secure energy insecure group is $37.0 \%$ and $38.5 \%$, which is much lower than that of both insecure group $(63.7 \%)$ and energy secure FCS insecure group $(62.0 \%)$. The poultry and meats FCS contribution rate of both secure group and FCS secure energy insecure group is $18.4 \%$ and $18.5 \%$, which is much higher than that of both insecure group (4.6\%) and energy secure FCS insecure group $(4.7 \%)$

\section{CONCLUTION}

The indicators of calorie intake and Food Consumption Score (FCS) developed by WFP were both effective indicators to measure food security, especially for the food access dimension. FCS has its own advantages for food security analysis. First of all, the raw data is the number of days consumption of each kind of foods in the previous 7 days, which is easy to collected and not easy to cause errors. Second, the data collection takes a very short time and cost less money. Third, the data processing work is easy and does not require complicated conversions. But at the same time, FCS also has its limitations in food security analysis. The most obvious one is that the number of days of consumption could not accurately reflect the real food consumption levels of households. For example, assume there are two households; both of them eat rice every day. One has enough rice to eat every day, but another could only eat very small amount of rice which far from the amount required. However, the score of rice consumption of these two households would be the same when using FCS to measure the food security, which is obviously not realistic.

Comparing with FCS, calorie consumption is a more direct indicator to measure food security. Although the calorie intake indicator is a direct indicator and easy to compare, but it still has its obvious limitations: on the one hand, the food consumption survey for nutrition analysis is time consuming, expensive, and the accuracy of the data is low; on the other hand, for rural residents in China, balanced diet is more worthy of concern. If calorie intake is the only indicator that used to measure the food security, then it seems too simple, and probably unable to locate people who are in poor diet quality. In this regard, FCS is superior to the calorie intake indicator.

The food contributions on calorie and FCS showed similar trends, indicating consistency. But when considering the energy secure, FCS insecure 
group and the FCS secure energy insecure group, FCS indicator narrow down the gap of grain contributions and enlarge the gap of poultry and meats contributions compare with the calorie indicator, which is exactly the effect of weights in FCS. Obviously, FCS considers diet diversity and balance more.

\section{REFERENCES}

[1] Babatunde R. O. and Qaim M. 2010. Impact of Off-farm Income on Food Security and Nutrition in Nigeria, Food Policy, 35(4): 303-311.

[2] Dasgupta P. 1993. An Inquiry into Well-being and Destitution. Clarendon Press, Oxford.

[3] FAO Statistic Division, 2012. Food Security Statistics from National Household Surveys.

[4] Gentilini U. and Webb P. 2008. How are We Doing on Poverty and Hunger Reduction? Food Policy, 33: 521532.

[5] Hoddinott J., and Yohannes Y., 2002. Dietary Diversity as a Food Security Indicator, FCND Discussion Paper No. 136, IFPRI. International Food Policy Research Institute, Washington, D. C.

[6] IFRRI Discussion Paper 00870, 2009. Validation of the World Food Programme's Food Consumption Score and Alternative Indicators of Household Food Security.
[7] Lintelo D. J. H. 2012. Measuring Political Commitment to Reducing Hunger and Under-nutrition: can it be done and will it help?, IDS Bulletin, 43(S1): 65-73.

[8] Masset E. 2011. A Review of Hunger Indices and Methods to Monitor Country Commitment to Fighting Hunger, Food Policy, 36: S102-S108.

[9] Morris S. Measuring nutritional dimensions of household food security. Methods for Rural Development Projects. Washington D. C.: International Food Policy Research Institute, 2001

[10] Nie Fengying, Amit Wadhwa, Wang Weijing. Analysis on Food Security and Vulnerability in Six Counties in Rural China. CFSN pub-lication NO. CN-07-1-11-01-A. China Agricultural Sciences and Technology Press: 2011.

[11] Qaim M., Stein A. J., and Meenakshi J. V. 2007. Economics of Biofortification, Agricultural Economics, 37(S1): 119-133.

[12] Svedberg P. 2000. Poverty and Undernutrition. Oxford University Press, New York.

[13] Svedberg P. 2011. How Many People are Malnourished? Annual Review of Nutrition, 31: 263-283.

[14] Walker S. P., Wachs T. D., Gardner J. M., Lozoff B., Wasserman G. A., Politt E., and Carter J. A. 2007. Child Development: risk factors for adverse outcomes in developing countries, The Lancet, 369(9556): 145-157.

[15] WFP. Emergency Food Security Assessment Handbook. 2005. 